\title{
TDMA Scheduling Design of Multihop Packet Radio Networks Based on Latin Squares
}

\author{
Ji-Her Ju \\ ZyXEL Communications Corp. \\ Science-Based Industrial Park \\ Hsinchu, Taiwan 300
}

\author{
Victor O.K. Li \\ Dept. of Electrical \& Electronic Engineering \\ The University of Hong Kong \\ Hong Kong, China
}

\begin{abstract}
Many transmission scheduling algorithms have been proposed to maximize the spatial reuse and minimize the Time Division Multiple Access (TDMA) frame length in multihop packet radio networks. Almost all existing algorithms assume exact network topology information and require recomputations when the network topology changes. In addition, existing work focuses on single channel TDMA systems. In this paper, we propose a multichannel topology-transparent algorithm based on latin squares. Our algorithm has the flexibility to allow the growth of the network, i.e., the network can add more mobile nodes without recomputation of transmission schedules for existing nodes. At the same time, a minimum throughput is guaranteed. We analyze the efficiency of our algorithm, and examine the topology-transparent characteristics and the sensitivity on design parameters by simulation.
\end{abstract}

\section{INTRODUCTION}

A radio network consists of a number of geographically dispersed radio units which communicate with each other. Due to limited transmission power, it may be necessary to relay a packet over multiple radio units to reach the destination. One important issue in this multihop mobile radio network is the design of transmission schedules [1]. A proper design not only guarantees successful information exchanges among radio units in the presence of conflicts but also maximizes the system throughput and minimizes the packet delay. In this context, we will focus on the scheduling problem in a Time Division Multiple Access (TDMA) network with multiple frequency channels called a multichannel TDMA network. Each radio unit in a multichannel TDMA network is equipped with a single transmitter and multiple receivers. So each radio unit can only transmit one packet but receive multiple packets in one slot simultaneously.

In such a multichannel TDMA network, the transmission time on each channel is divided into time slots, which are in turn grouped into frames. The frames on all the channels are synchronized with each other. The time aligned frames on all of the channels are grouped into a multichannel TDMA frame. Each slot is designed to accommodate the transmission time of one fixed size packet and the guard time, corresponding to the maximum differential propagation delay between any pair of radio units in the network. Two types of communication conflicts on a single channel may arise [3]. The first type of conflict, called a primary conflict, occurs when a radio unit, transmitting in a given slot, is receiving a packet in the same slot on the same channel. This also implies the converse: a receiving radio unit cannot be transmitting on the same channel at the same time. The other conflict, referred to as a secondary conflict, happens if a radio unit receives more than one packet in a slot on the same channel. In both cases, all packets are rendered useless.
Previous studies [1], [5], [6], [7], [9], [10] on transmission scheduling algorithm concentrated on designing fair conflictfree algorithms which maximize the system throughput and minimize the frame length by using graph theory [6], [11]. Most algorithms are centralized, i.e., they need global network connectivity information to achieve their goals. Ephremides and Truong [5] proposed a distributed algorithm which requires using connectivity information up to two hops. The distributed algorithm of Ramaswami and Parhi [10] only needs one-hop connectivity information. As one can expect, however, changes of network topology due to the movements of mobile radio units may render an optimal design obsolete. Therefore, the efficiency and robustness of the above algorithms disappear in mobile networks. By allowing partial contentions, Chlamtac and Farago [3] developed an algorithm which is topology transparent. However, the throughput of their algorithm is lower than a conventional TDMA scheme for some cases. Following their approach, but with a different design strategy, Ju and Li [8] proposed another topology transparent algorithm which is optimal in that it ma:imizes the guaranteed throughput. They also showed that their algorithm is insensitive to the design parameters.

The existing work on TDMA scheduling has focused on single channel systems. This paper deals with multichannel systems. Multichannel TDMA systems are natural extensions of single channel TDMA systems. With a fixed total bandwidth or channel resource, a multichannel TDMA system outperforms a single channel TDMA system in the following aspects [2]:

(1) Multichannel TDMA systems usually have better reliability than single channel TDMA systems.

(2) Individual channels operate at a lower rate, and synchronization is easier in multichannel TDMA systems.

(3) Multichannel TDMA systems have more flexibility to allow system growth by adding more channels.

Since single channel algorithms only attempt to optimize the transmission schedules on one channel, they cannot be easily extended to multichannel systems. A more efficient design in transmission schedules for multichannel systems is to treat all the transmission slots on all channels as a two-dimensional (time and frequency) transmission scheduling problem.

In this paper, a scheduling algorithm for multichannel TDMA networks based on latin squares is proposed. Using this algorithm, every radio unit decides its own transmission schedule before it participates in network operations. Although this algorithm does not require central control or information exchanges 
between neighboring network nodes, it enjoys very good delay characteristics. The performance is not seriously degraded by dynamic changes of network topology even though transmission schedules are not recomputed.

Some background and definitions related to the algorithm will be given in Section 2. In Section 3, the operation concepts are presented and some characteristics of the algorithm are analyzed to decide the operation parameters for a given network. Then an approximate discrete time queuing model is proposed in Section 4. In Section 5, some numerical and simulation results are shown to validate our analytical model and to evaluate the performance of our algorithm. Finally, we conclude in Section 6.

\section{MODEL AND DEFinitions}

The total number of available channels in our multichannel TDMA system is assumed to be $M$. Each radio unit has $M$ receivers such that it can receive packets on all channels. But it can transmit at most one packet at a time by tuning its synthesizer to the appropriate frequency of a chosen channel. On the same channel, a radio unit cannot transmit and receive simultaneously. The time axis of each channel is divided into slots and is synchronous with other channels. The transmission channel is assumed to be error-free and reception failure is due only to packet collisions. The acknowledgments are also assumed error-free and instantaneous.

The maximum total number of mobile radio units $(N)$ and the maximum number of neighbors of any radio unit $\left(D_{\max }\right)$ are known to the network designer. Based on these two parameters and by making use of the special characteristics of latin squares, a topology-transparent scheduling algorithm will be developed such that every radio unit can retain a pre-determined minimum throughput no matter how the topology changes if the actual values of $N$ and $D_{\max }$ do not deviate significantly from the design values.

The following definitions and theorems [4] are useful in the development of our algorithm.

Definition 1: An $m \times n$ rectangular array formed by the symbols $1,2, \ldots, k$, where $k \geq m$ and $k \geq n$, is called a latin rectangle if every symbol appears at most once in each row and once in each column.

When the number of rows, columns and symbols are the same, we call it a latin square, defined as follows:

Definition 2: A latin square of order $n$ is an $n \times n$ square array composed of $n$ symbols from 1 to $n$ such that each symbol appears once in each row and once in each column.

The square arrays $A$ and $B$ shown below are examples of latin squares of order 4 .

$$
A=\left[\begin{array}{llll}
1 & 2 & 3 & 4 \\
2 & 1 & 4 & 3 \\
3 & 4 & 1 & 2 \\
4 & 3 & 2 & 1
\end{array}\right], \quad B=\left[\begin{array}{llll}
4 & 1 & 2 & 3 \\
3 & 2 & 1 & 4 \\
1 & 4 & 3 & 2 \\
2 & 3 & 4 & 1
\end{array}\right]
$$

Definition 3: Two distinct $n \times n$ latin squares $A=\left(a_{i, j}\right)$ and $B=\left(b_{i, j}\right)$, where $a_{i, j}$ and $b_{i, j} \in\{1,2, \ldots, n\}$, are said to be orthogonal if the $n^{2}$ ordered pairs $\left(a_{i, j}, b_{i, j}\right)$ are all different.

Thus, the two $4 \times 4$ latin squares $A$ and $B$ shown above are orthogonal. More generally, if $A^{(1)}, A^{(2)}, \ldots, A^{(r)}$ are distinct latin squares, they are said to form an orthogonal family if every pair of them is orthogonal.

Theorem 1: If there is an orthogonal family of $r$ latin squares of order $n$, then $r \leq n-1$.

Definition 4: If an orthogonal family of latin squares of order $n$ has size $(n-1)$, i.e., the number of latin squares in the orthogonal family is $(n-1)$, it is called complete.

Theorem 2: If $n>1$ and $n=p^{k}$, where $p$ is a prime number and $k$ is a positive integer, then there is a complete orthogonal family of latin squares of order $n$.

The proofs of Theorems 1 and 2 can be found in [4].

\section{SCheduling Algorithm}

Consider a multichannel TDMA network with $M$ channels. The multichannel TDMA frames are constructed as an $M \times p$ table, where $p$ is the number of slots in a frame on each channel. During each frame, every radio unit may be assigned $M$ slots to transmit its packets according to its unique time slot assignment pattern. These time slot assignment patterns are constructed from a corresponding orthogonal family of $p \times p$ latin squares. Basically $p$ radio units share one common $p \times p$ latin square from this orthogonal family, and the time slot assignment pattern of each of these $p$ radio units is represented by one of the $p$ distinct symbol patterns in the latin square.

Assume two radio units, say radio units $u$ and $v$, are assigned symbol $a$ in latin square $A$ and symbol $b$ in latin square $B$, respectively. So, the pattern of symbol $a$ in latin square $A$ corresponds to the time slot assignment pattern of radio unit $u$ in the multichannel TDMA frames. Similarly, the pattern of symbol $b$ in latin square $B$ corresponds to the time slot assignment pattern of radio unit $v$. For example, let $a=2$ and $A$ is the square shown in section 2 , then the first slot on channel 2 , the second slot on channel 1, the third slot on channel 4 , and the fourth slot on channel 3 will be assigned to radio unit $u$. Here the channels correspond to the rows and the time slots correspond to the columns. If $b=3$ and $B$ is defined as in section 2 , then the first slot on channel 2 , the second slot on channel 4 , the third slot on channel 3, and the fourth slot on channel 1 are the transmission slots of radio unit $v$. In this example, we see that the ordered pair $(2,3)$ of squares $A$ and $B$ occurs at the second row, first column. This means that if both radio units $u$ and $v$ transmit at the first slot on channel 2 , then they will collide.

For generality, we have the following definition and theorem to describe the collision and collision-free conditions.

Definition 5: Assume that the transmission schedule, i.e., the time slot assignment pattern, of each radio unit is determined by the pattern of a symbol in a latin square. Let two radio units, $s$ and $d$, be assigned symbols $a$ and $b$ in two $p \times p$ orthogonal latin squares $A$ and $B$, respectively. If the ordered pair $(\mathrm{a}, \mathrm{b})$ 
of squares $A$ and $B$ occurs at the $i$-th row, $j$-th column, i.e., $\left[A_{i, j}\right]=a$ and $\left[B_{i, j}\right]=b$, we say that there is a chance of collision at the $j$-th slot on channel $i$ for radio units $s$ and $d$.

Theorem 3: If two radio units are assigned two distinct symbols in a common latin square, these two radio units will be collision free with each other all the time. If they are assigned symbols from two different orthogonal latin squares, there is at most one collision for these two radio units in every multichannel TDMA frame.

Proof: The first part of the theorem follows directly from the definition of a latin square. Because every symbol only appears once in each row and once in each column, any two time slot assignment patterns chosen from the same latin square will not have any overlap in their patterns, i.e., they do not have any chance to collide with each other. The second part follows from definition 3. Hence, the ordered pair $(a, b)$ for any two different latin squares, where $a$ and $b \in\{1,2, \ldots, n\}$, can only appear once. This implies that these two radio units will only have one chance for collision.

Theorem 4: In a given network, each radio unit has a transmission schedule corresponding to a symbol pattern in one of the latin squares from an order $p$ orthogonal latin square family. Consider any radio unit surrounded by $D$ other radio units, it suffers from at most $D$ collisions with the other $D$ radio units. And, the minimum number of collision chances that it can have is equal to $\max (D+1-p, 0)$.

Proof: From Theorem 3, we know that each neighbor can cause at most one collision to a specific radio unit. For a particular radio unit, say radio unit $s$, in the worst case, the other $D$ radio units are all neighboring radio units, and their transmissions follow latin squares which are different from the latin square chosen by radio unit $s$. Hence, the maximum number of collision chances for radio unit $s$ is $D$. To calculate the minimum number of collision chances, we have to count the maximum number of radio units that can determine their transmission schedules from a common latin square, which is $p$. For these $p$ radio units, no collision will occur among them according to Theorem 3. From the same theorem, we know that there is only one collision chance for any two radio units belonging to different orthogonal latin squares. So, each of the remaining $\max (D-(p-1), 0)=\max (D+1-p, 0)$ radio units will contribute one collision to the specific radio unit because they belong to different orthogonal latin squares.

Consider a multichannel TDMA network with $M$ TDMA channels and $N$ mobile radio units. The maximum number of neighbors of a radio unit in this network is $D_{\max }$. We assume that the size of the orthogonal family of $p \times p$ latin squares is $r$. The transmission schedule of each radio unit is determined by one of the $p$ distinct symbol patterns in a $p \times p$ orthogonal latin square. When $p>M$, one $p \times p$ latin square can be trimmed into an $M \times p$ latin rectangle. Each latin square or latin rectangle can be used to construct the transmission schedules for $p$ different radio units. To make sure every radio unit has a unique transmission schedule associated with these latin squares or latin rectangles, the following equation must be sat- isfied:

$$
p \times r \geq N .
$$

From Theorem 3, we know that the number of collision chances in each multichannel TDMA frame for any two radio units is either 0 or 1 . The maximum number of neighbors of a radio unit is $D_{\max }$ and each radio unit will be assigned $\min (M, p)$ transmission slots in each multichannel TDMA frame. Every radio unit will have its non-zero guaranteed throughput when the following inequality holds:

$$
p \geq T_{\max } \geq T \geq T_{\min }>0 .
$$

where $T$ is the number of successful transmissions in each frame for every radio unit; $T_{\max }$ and $T_{\min }$ are the upper and the lower bounds of $T$, respectively, and

$$
\begin{aligned}
T & =\min (M, p)-(\text { number of collisions in a frame) } \\
T_{\max } & = \begin{cases}p-\max \left(D_{\max }+1-p, 0\right), & \text { if } \mathrm{p} \leq \mathrm{M} \\
M-\max \left(D_{\max }+1-p, 0\right), & \text { if } \mathrm{p}>\mathrm{M} .\end{cases}
\end{aligned}
$$

and

$$
T_{\min }= \begin{cases}p-D_{\max }, & \text { if } \mathrm{p} \leq \mathrm{M} \\ M-D_{\max }, & \text { if } \mathrm{p}>\mathrm{M}\end{cases}
$$

Hence, to ensure that every radio unit has non-zero guaranteed throughput, $p$ has to be greater than $D_{\max }$ when $p \leq M$ or $M$ has to be greater than $D_{\max }$ when $p>M$. In order to evaluate the performance, we define $G_{L}$ as the guaranteed throughput (lower bound) of the TDMA scheduling algorithm as follows:

Definition 6: The guaranteed throughput $G_{L}$ is defined as the ratio of the number of guaranteed successful transmissions in each frame to the frame length $L$, i.e.,

$$
G_{L}=T_{\min } / L, \quad \text { where } L=p .
$$

Similarly, we define the best throughput (upper bound) of the TDMA scheduling algorithm as follows:

Definition 7: The best throughput $G_{U}$ is defined as the ratio of the maximum number of successful transmissions in each frame to the frame length $L$, i.e.,

$$
G_{U}=T_{\max } / L, \quad \text { where } L=p .
$$

Theorem 5: For given $D_{\max }, N$ and $M$, the maximal nonzero upper and lower bounds of throughput $G$ are as follows:

$$
\begin{aligned}
& 1 \geq G \geq 1-\left(D_{\max } / M\right), \quad \text { if } \mathrm{p} \leq \mathrm{M} \\
& M, \quad M,[N / r]) \geq G \geq \frac{M-D_{\max }}{\max (M,[N / r])}, \quad \text { if } \mathrm{p}>\mathrm{M} \text {. }
\end{aligned}
$$

Proof: When $p \leq M$, from (6) and (7) we know the upper and lower bounds of $G$ are

$$
\begin{aligned}
G_{U} & =T_{\max } / L \\
& =\left[p-\max \left(D_{\max }+1-p, 0\right)\right] / p \\
& =1-\left[\max \left(D_{\max }+1-p, 0\right) / p\right]
\end{aligned}
$$

and

$$
\begin{aligned}
G_{L} & =T_{\min } / L \\
& =\left(p-D_{\max }\right) / p \\
& =1-D_{\max } / p
\end{aligned}
$$


From the above two equations, we can see that the two bounds will increase with $p$. To ensure that the minimum throughput is greater than zero and every radio unit has its own unique transmission schedule, $p$ has to be greater than $D_{\max }$ and no less than $\lceil N / r\rceil$. We can get $\max \left(D_{\max }+1-p, 0\right)=0$ and $\lceil N / r\rceil \leq p \leq M$. Hence, the maximal upper and lower bounds of throughput are

$$
G_{U}=1
$$

and

$$
G_{L}=1-\left(D_{\max }\right) / M
$$

when $p=M$.

Similarly, if $p>M$, the non-zero upper and lower bounds of $G$ are

$$
\begin{aligned}
G_{U} & =T_{\max } / L \\
& =\left[M-\max \left(D_{\max }+1-p, 0\right)\right] / p \\
& =M / p
\end{aligned}
$$

and

$$
\begin{aligned}
G_{L} & =T_{\min } / L \\
& =\left(M-D_{\max }\right) / p
\end{aligned}
$$

These two bounds will decrease when $p$ increases. Combining $p>\lceil N / r\rceil$ and $p>M$, we can get $p>\max (M,[N / r\rceil)$. So, the maximal upper and lower bounds are

$$
G_{U}=M / \max (M,\lceil N / r\rceil)
$$

and

$$
G_{L}=\left(M-D_{\max }\right) / \max (M,\lceil N / r\rceil)
$$

when $p=\max (M,\lceil N / r\rceil)$.

In the above theorem, $M \geq p$ corresponds to the number of available channels greater than the number of transmission slots assigned to a radio unit in a multichannel TDMA frame for the given network architecture ( $N$ and $D_{\max }$ are known). In this case, the guaranteed throughput $\left(G_{L}\right)$ can be maximized when we choose $p$ equal to the maximum number of available channels. Since the number of available channels in the network must be limited, $M<p$ will occur for some given $N$ and $D_{\max }$ values. In these cases, the upper and lower bounds of the guaranteed throughput may be affected by the size of the orthogonal latin squares family. A larger value of $r$ may make both the upper and lower bounds of guaranteed throughput larger which is preferred from the system designer's point of view. From Theorem 1 and Definition 4, we know the size of an orthogonal latin squares family will be maximized when the orthogonal latin squares family is complete.

Based on the above theorems, the optimal scheduling algorithm will be constructed as follows.

\section{Optimal Algorithm:}

- Use Theorem 5 to determine the optimal integer of $p$ for the given $M, N$ and $D_{\max }$ such that $G_{U}$ and $G_{L}$ are maximized.
- Randomly assign a symbol in a latin square or latin rectangle to each radio unit.

- Each radio unit constructs its transmission schedule from its unique symbol pattern in its latin square or latin rectangle and transmits its packets according to its transmission schedule.

Here, we assign the symbols in latin squares to radio units randomly, and each radio unit only uses one symbol pattern to construct its transmission schedule. If we know that some radio units are always adjacent to each other, then we can try to assign different symbols in a common latin square or latin rectangle to these radio units to reduce the number of collisions among them. For some particular radio units with predictably heavy traffic, we can also assign multiple different symbols in latin squares or latin rectangles to it to increase its throughput. For the cases of $D_{\max }>M$, to ensure each radio unit has non-zero guaranteed throughput, the transmission schedules can also be constructed by using a similar scheme, but with larger frame size $\left(\left\lceil\frac{D_{\max }}{M}\right\rceil \times p\right.$ slots $)$

\section{ANALytical MODEL}

In this section, an approximate discrete time $\mathrm{M} / \mathrm{M} / 1$ queuing model [12] with bulk arrivals is proposed to evaluate the average packet delay in a multichannel TDMA system using the algorithm introduced in the previous section. We assume that arrivals occur just after the beginning of a slot, departures take place just before the end of a slot, and each radio unit has infinite buffer. Each radio unit with a packet to transmit chooses the transmission channel randomly. Note that the latter is an approximation since the radio unit will actually choose its transmission channel according to the latin square method. The number of arrivals in each slot are assumed to be a sequence of independent, identically distributed (i.i.d.) random variables with a specific distribution. The probability of $k$ arrivals in a slot is given by

$$
a_{k}=\operatorname{Pr}(\text { number of arrivals in a slot }=k) \text {. }
$$

The state transition diagram of our queuing model is shown in Figure 1, where the state index represents the number of packets stored in the radio unit and $p_{i, j}$ is the transition probability from state $i$ to state $j$. The transmission of a packet is successful when it is not involved in any collision, i.e., it is successfully received by its destination. The probability of successful transmission is denoted by $\mu$, which corresponds to the service rate of the system for each radio unit. Let the equilibrium probability for the number of packets stored in a radio unit be $\left\{q_{k}, k=0,1,2, \ldots\right\}$.

In general, the transition probabilities can be specified as

$$
\begin{gathered}
p_{i, j}=\operatorname{Pr}(j \text { packets are queued in slot } t+1 \mid i \\
\text { packets were queued in slot } t) \\
= \begin{cases}a_{j} & \text { if } i=0, \\
0 & \text { if } i>0 \text { and } j<i-1, \\
a_{0} \mu & \text { if } i>0 \text { and } j=i-1, \\
a_{j+1-i} \mu+a_{j-i}(1-\mu) & \text { otherwise. }\end{cases}
\end{gathered}
$$


The balance equation for this discrete Markov chain is

$$
\begin{aligned}
q_{k} & =q_{k} p_{k, k}+q_{k+1} p_{k+1, k}+\sum_{i=1}^{k-1} q_{i} p_{i, k}+q_{0} p_{0, k} \\
& =\sum_{i=1}^{k+1} q_{i}\left[a_{k+1-i} \mu+a_{k-i}(1-\mu)\right]+q_{0} a_{k} .
\end{aligned}
$$

Hence the $z$-transform of the queue length is

$$
\begin{aligned}
Q(z) & =\sum_{k=0}^{\infty} q_{k} z^{k} \\
& =\frac{q_{0} A(z)[z-D(z)]}{z-A(z) D(z)}
\end{aligned}
$$

where $A(z)=\sum_{k=0}^{\infty} a_{k} z^{k}$ and $D(z)=\mu+(1-\mu) z$. Substituting $z=1$ into (21), and noting that $\mathrm{A}(1)=1$, an indeterminacy of the form $0 / 0$, can be resolved using L'Hospital's rule, we get

$$
q_{0}=1-\frac{A^{\prime}(1)}{\mu}
$$

The average queue length can be obtained from the $\mathrm{z}$-transform of the queue length as

$$
\begin{aligned}
N_{q} & =\sum_{k=0}^{\infty} k q_{k} \\
& =Q^{\prime}(1) \\
& =A^{\prime}(1)+\frac{A^{\prime \prime}(1)+2 A^{\prime}(1)(1-\mu)}{2\left[\mu-A^{\prime}(1)\right]} .
\end{aligned}
$$

Using Little's result, we can find the average time each packet spends in the system as

$$
\begin{aligned}
W_{s} & =\frac{N_{q}}{A^{\prime}(1)} \\
& =1+\frac{A^{\prime \prime}(1)+2 A^{\prime}(1)(1-\mu)}{2 A^{\prime}(1)\left[\mu-A^{\prime}(1)\right]}
\end{aligned}
$$

where $A^{\prime}(1)=$ the average number of arrivals per slot at each mobile user.

For a specific radio unit, say radio unit $d$, it is surrounded by $D$ other radio units. The packet transmitted from a neighboring radio unit $s$ is successfully received by radio unit $d$ if radio unit $d$ and the other $D-1$ neighboring radio units of radio unit $d$ do not transmit in the same slot on the same channel as radio unit $s$. Suppose $X$ radio units are transmitting packets in the same slot as radio unit $s$. The probability of this event is

$$
\begin{gathered}
\operatorname{Pr}(X=i)=\operatorname{Pr}(i \text { simultaneously transmitted packets } \\
\text { in the same slot as radio unit s) } \\
=\left(\begin{array}{c}
D \\
i
\end{array}\right) \rho^{i}(1-\rho)^{D-i}\left(\frac{\min (M, p)}{p}\right)^{i} \\
i=0,1, \ldots, D
\end{gathered}
$$

where $\rho$ is the utilization factor.
Let $Y$ out of these $X$ active radio units decide their transmission schedules from the same latin square as radio unit $s$.

$\operatorname{Pr}(j$ out of $i$ mobile radio units select symbol patterns from the same latin square as radio unit $s$ )

$=\operatorname{Pr}(Y=j \mid X=i)$

$=\frac{\left(\begin{array}{c}p-1 \\ j\end{array}\right)\left(\begin{array}{c}K-p \\ i-j\end{array}\right)}{\left(\begin{array}{c}K-1 \\ i\end{array}\right)}, i=0,1, \ldots, D$ and $j=0,1, \ldots, i$

where $K=p \times r$ is the total number of symbol patterns in the orthogonal latin square family, $p$ is the order of the chosen latin squares, and $r$ is the size of the orthogonal latin square family.

These $Y$ radio units will not cause any collisions at radio unit $d$, but the other $X-Y$ neighbors may collide with the transmission from radio unit $s$ to radio unit $d$ on the same channel.

$$
\begin{aligned}
& \operatorname{Pr}(\text { transmission collision } \mid Y=j \text { and } X=i) \\
= & 1-\operatorname{Pr}(\text { successful transmission } \mid Y=j \text { and } X=i) \\
= & 1-\left(\frac{\min (M, p)-1}{\min (M, p)}\right)^{i-j}
\end{aligned}
$$

where $M$ is the number of channels in the multichannel TDMA system, and $\min (M, p)$ is the number of transmission slots in each multichannel TDMA frame for each radio unit.

Hence, the probability of a successful packet transmission from radio unit $s$ to radio unit $d$ is

$$
\begin{aligned}
& \mu=\operatorname{Pr}(\text { a succ. pkt. transm. from } s \text { to } d) \\
&=\sum_{i=0}^{D} \sum_{j=0}^{i} \operatorname{Pr}(X=i, Y=j) \times \\
&=\sum_{i=0}^{D} \sum_{j=0}^{i} \operatorname{Pr} \text { (succ. trans. } \operatorname{Pr}(Y=j \mid X=i) \operatorname{Pr}(X=i) \times \\
&=\sum_{i=0}^{D} \sum_{j=0}^{i} \frac{\operatorname{Pr}\left(\begin{array}{c}
D \\
i
\end{array}\right)\left(\begin{array}{c}
p-1 \\
j
\end{array}\right)\left(\begin{array}{c}
K-p \\
i-j
\end{array}\right)}{\left(\begin{array}{c}
K-1 \\
i
\end{array}\right)} \rho^{i}(1-\rho)^{D-i} \times \\
&\left(\frac{\min (M, p)}{p}\right)^{i}\left(\frac{\min (M, p)-1}{\min (M, p)}\right)^{i-j}
\end{aligned}
$$

Substituting

$$
\begin{aligned}
q_{0} & =\operatorname{Pr}(\text { mobile radio unit has nothing to send }) \\
& =1-\rho
\end{aligned}
$$

into (22), we can get

$$
\rho=\frac{A^{\prime}(1)}{\mu} .
$$

Solving (28) and (30), we can have the probability of successful transmission $\mu$. Obviously, substituting the value of $\mu$ into (24), we can find the average system delay for a packet, $W_{s}$, 
which is defined as the number of time slots between the packet generation and the completion of its successful transmission.

For the case of Poisson arrival, the z-transform of the number of packets arriving in a slot is

$$
A(z)=e^{\lambda(z-1)}
$$

where $\lambda$ is the average number of arrivals in each slot. From (24), the average packet delay will be

$$
W_{s}=\frac{2-\lambda}{2(\mu-\lambda)} .
$$

Similarly, for the case of Bernoulli arrivals, the z-transform of the number of packets arriving in a slot is

$$
A(z)=(1-\alpha)+\alpha z
$$

where $\alpha$ is the probability for a packet arriving in a slot. So, the the average packet delay is

$$
W_{s}=\frac{1-\alpha}{\mu-\alpha}
$$

\section{PERFormanCE ANALYSIS}

In the previous section, an approximate analytical model for evaluating the average packet delay of the multichannel TDMA system with bulk arrival traffic is proposed. To validate this analytical model, some simulation results will be given. In the simulation model we again assume that each queue has infinite capacity and uses First-Come-First-Served (FCFS) service discipline. Every radio unit can only transmit one packet in each slot. The destination of a packet is randomly chosen from its neighbors but the transmission channel is assigned deterministically using our algorithm. The acknowledgment is assumed instantaneously received and is error free. Two different traffic models, Poisson arrivals and Bernoulli arrivals, were chosen for comparison. For Poisson arrivals, the number of arrivals in each slot are Poisson random variables and its average value is $\lambda$. In Bernoulli arrivals, a packet will arrive in a slot with probability $\alpha$.

First, to show the accuracy of our approximate analytical model, we compare the simulation and analytical results for Poisson arrivals and Bernoulli arrivals with $D=2, p=4$ and $M=4$ in Figures 2 and 3, respectively. From these figures, we find that our approximate analytical results match very well with the simulation results for both Poisson arrivals and Bernoulli arrivals. To evaluate the effect of topology changes on the delay, we generate simulation results for two different types of networks, namely, a highly mobile one in which the topology changes every 1000 slots, and a low mobility network in which the topology changes every 10000 slots. The results in Figures 2 and 3 show that the delay varies very little with the frequency of topology changes. This proves that our algorithm is very robust to topology changes. Our algorithm is mainly developed based on the system parameter $D$, which is the number of neighbors per radio unit in the network. In real mobile networks, this parameter may vary with time. In Figures 4 and 5, we see that the delay does not increase much at low traffic although the actual value of $D$ exceeds the design value of $D$. So if the network is not operating at high traffic conditions, the inaccuracy of the estimated value of $D$ is acceptable in our algorithm.

\section{CONCLUSIONS}

In a multihop TDMA network, scheduling transmission is vital. To design a scheduling algorithm which is topology transparent is particularly important when the network is highly mobile. We have designed a topology transparent scheduling algorithm for multichannel TDMA systems based on latin squares. This algorithm can provide non-zero maximal guaranteed throughput to each radio unit in the network.

We derived some useful bounds of the throughput of our algorithm, and the efficiency of our algorithm has been examined analytically. In addition, the topology-transparent feature has been proven by simulation. The delay is not sensitive to the accuracy of the estimated value of $D$ at low traffic situation.

\section{REFERENCES}

[1] D. J. Baker and A. Ephremides. The architectural organization of a mo bile radio network via a distributed algorithm. IEEE Trans. on Commun., 29:1694-1701, November 1981.

[2] J. F. Chang and T. H. Yang. Multichannel ARQ protocols. IEEE Trans. on Commun., 41:592-598, April 1993.

[3] I. Chlamtac and A. Farago. Making transmission schedules immune to topology changes in multi-hop packet radio networks. IEEE/ACM Trans. on Networking, 2(1):23-29, Feb. 1994.

[4] J. Denes and A. D. Keedwell. Latin squares and their applications. Academic Press Inc. New York, 1974.

[5] A. Ephremides and T. V. Truong. Scheduling broadcasts in multihop radio networks. IEEE Trans. on Communications, 38(4):456-460, April 1990.

[6] B. Hajek and G. Sasaki. Link scheduling in polynomial time. IEEE Trans. on Information Theory, 34:910-917, September 1988.

[7] K. Hung and T. Yum. Fair and efficient transmission scheduling in multihop packet radio networks. In IEEE GLOBECOM '92, pages 6-10, 1992.

[8] J. H. Ju and V. O. K. Li. An optimal topology-transparent scheduling method in multihop packet radio networks. IEEE/ACM Trans. on Networking, 6(3):298-306, June 1998.

[9] A. Kershenbaum and M. J. Post. Distributed scheduling of CDMA network with minimal information. IEEE Trans. on Commun., 39(1):17-20, Jan. 1991

[10] R. Ramaswami and K. K. Parhi. Distributed scheduling of broadcasts in a radio network. In IEEE INFOCOM'89, pages 497-504, 1989.

[11] L. Tassiulas and A. Ephremides. Jointly optimal routing and scheduling in packet radio networks. IEEE Trans. on Information Theory, 38:165-168, January 1992

[12] M. E. Woodward. Communication and computer networks - modeling with discrete-time queues. IEEE Computer Society Press, 1994. 


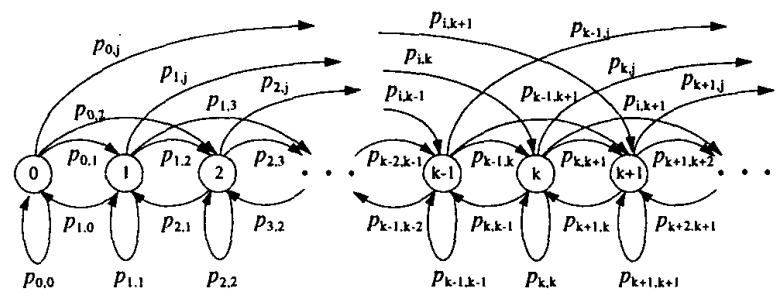

Fig. 1. The transition diagram for discrete time $M / M / 1$ queuing model with bulk arrivals.

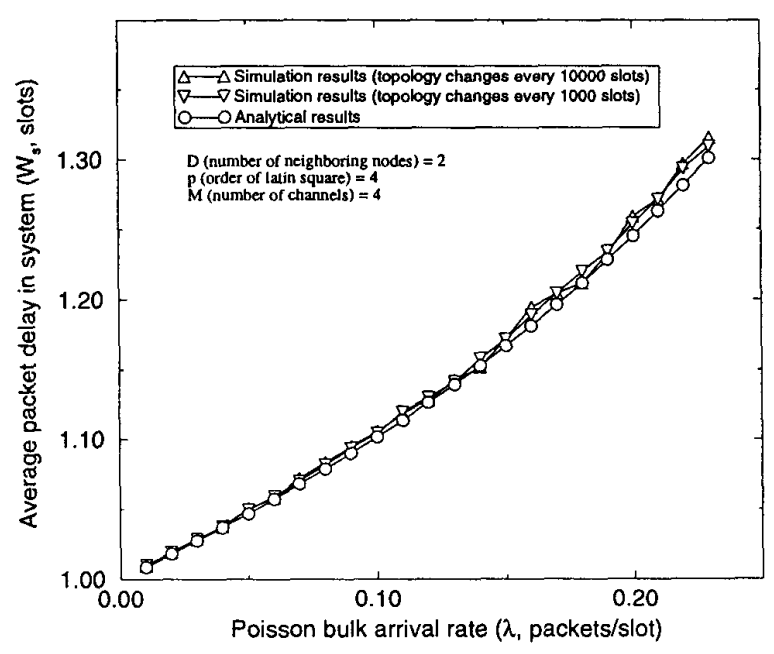

Fig. 2. The average packet delay in a system with Poisson bulk arrivals.

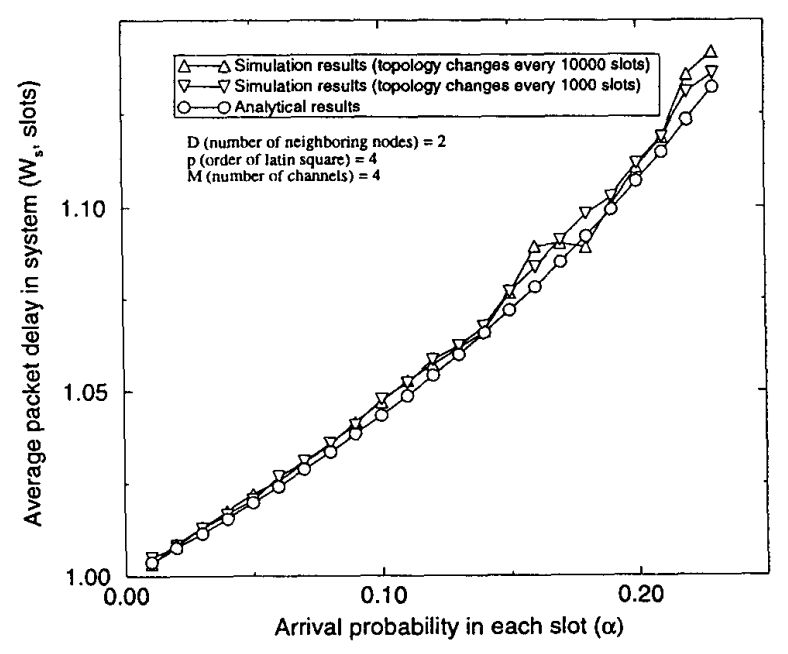

Fig. 3. The average packet delay in a system with Bernoulli arrivals.

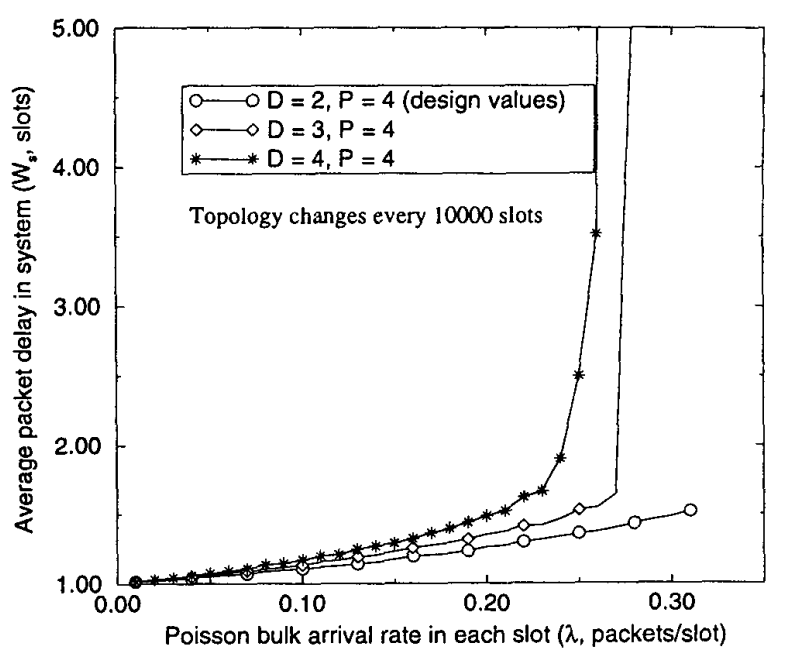

Fig. 4. The average packet delay in the system vs. the number of neighboring radio units (Poisson case).

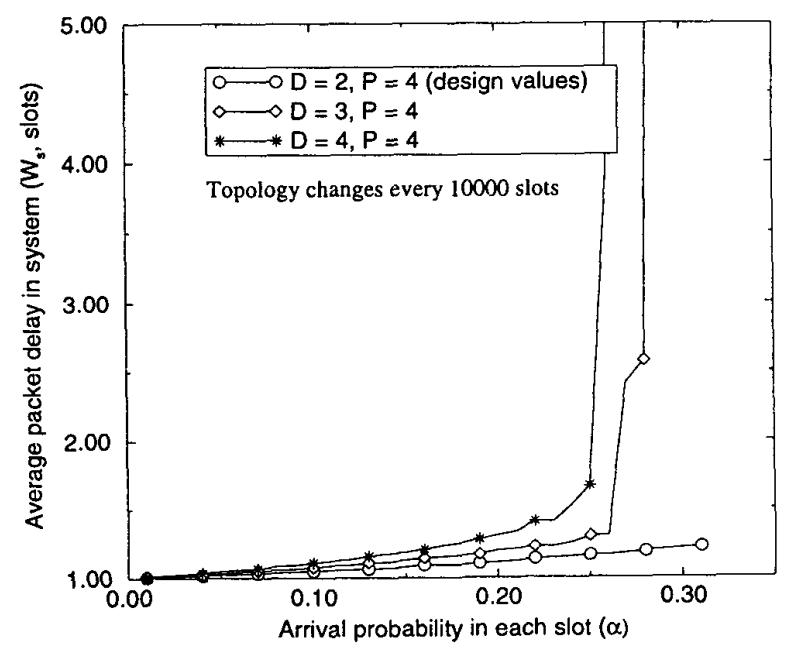

Fig. 5. The average packet delay in the system vs. the number of neighboring radio units (Bernoulli case). 